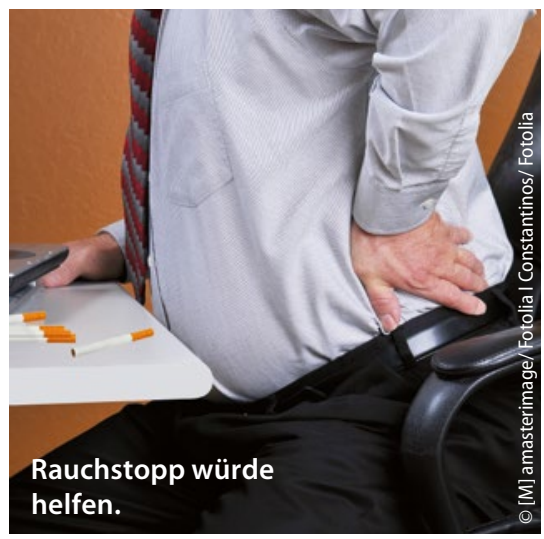

Wenig beachtetes Risiko

\title{
Mehr Ischialgien bei Rauchern
}

_ Wer raucht, setzt sich einem erhöhten Risiko aus, eine klinisch gesicherte Ischialgie zu entwickeln. Das legen die Ergebnisse einer Metaanalyse mit rund 480.000 Patienten nahe. Die Quotenverhältnisse lagen bei Rauchern um 64\% bzw. 35\% höher als bei Nichtrauchern. Die Wahrscheinlichkeit, wegen eines Bandscheibenprolapses ins Kranken- haus zu kommen oder operiert zu werden, war bei Rauchern ebenfalls signifikant erhöht (+45\%). Unter ehemaligen Rauchern war der Anteil von Ischialgiepatienten gegenüber Nichtrauchern nur noch geringfügig erhöht (+9\%).

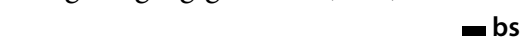

- Shiri Ret al. Amer J Med 2015, online 21. September

\section{Prospektiv untersucht}

\section{Wie sicher ist die Analgesie mit Cannabis?}

_ Bei der medizinischen Anwendung eines qualitätskontrollierten Cannabispräparates zur Schmerzlinderung ist nicht mit schweren Nebenwirkungen zu rechnen, wie eine kanadische Studie nahelegt. An der prospektiven 1-JahresStudie nahmen Patienten mit chronischen, nicht durch Krebs verursachten Schmerzen teil. 215 nutzten ein Cannabisprodukt mit einem Anteil von 12,5\% Tetrahydrocannabinol, das geraucht oder oral oder vaporisiert aufgenommen werden konnte. Die mediane Tagesdosis lag bei 2,5 g. Die Kontrollgruppe mit 216 Patienten verwendete kein Cannabis.

Im Gruppenvergleich gab es keinen signifikanten Unterschied in der Inzidenz schwerer unerwünschter Wirkungen. Am häufigsten waren in der Verumgruppe Bauchschmerzen, Obstipation und Neph- rolithiasis (jeweils drei Patienten), doch in keinem Fall konnte ein Zusammenhang mit dem Cannabisgebrauch sicher festgestellt werden. Lediglich leicht oder moderat ausgeprägte Nebenwirkungen traten in der Verumgruppe häufiger auf. Eine signifikante Reduktion der durchschnittlichen Schmerzintensität war bei Studienende nur in der Cannabisgruppe festzustellen.

- ple

\section{Mit bukkalem Opioid}

\section{Durchbruchschmerzen schneller unter Kontrolle}

_ Mit bukkal verabreichten Fentanyltabletten lassen sich Durchbruchschmerzen besser lindern als mit oral appliziertem Morphin, wie aus einer randomisierten Cross-over-Studie hervorgeht. An der Studie nahmen 81 Krebspatienten teil, von denen die meisten Urogenital-, Gastrointestinal- oder Lungentumoren hatten. Unter einer Analgesie, mit der sich die Schmerzintensität bei einem Wert von 4 auf einer Schmerzskala von 0 bis 10 für zwei Tage halten ließ, wurden die Patienten angehalten, dann um eine Therapie gegen Durchbruchschmerzen zu bitten, wenn die Schmerzen stärker wurden.
Im Zeitraum von drei Tagen erhielten die Patienten bukkales Fentanyl $(100 \mu \mathrm{g})$ oder orales Morphin im Cross-over-Design. Die Dosis lag bei Fentanyl oder 10 mg oralem Morphin, wenn sie zur Basisanalgesie bereits $60 \mathrm{mg}$ orales Morphinäquivalent erhalten hatten. Lag dieses bei $120 \mathrm{mg}$, wurde auch die Dosis von Fentanyl und oralem Morphin verdoppelt.

Durch beide Präparate wurde die Schmerzintensität signifikant reduziert ( $\mathrm{p}<0,0005) .15$ und 30 Minuten nach Applikation waren jedoch statistisch signifikante Differenzen festzustellen. So wurde eine Schmerzreduktion um mindestens 33\% (moderat) unter Fentanyl bei deutlich mehr der insgesamt über 260 dokumentierten Schmerzepisoden registriert als unter dem Vergleichspräparat (nach 15 Minuten: $76,5 \%$ versus 32,8\%; nach 30 Minuten: $89,0 \%$ versus $54,9 \% ; \mathrm{p}<0,0005)$. Ebenfalls signifikant waren die Unterschiede, wenn eine Schmerzreduktion um mindestens 50\% (substanziell) betrachtet wurde. Den Unterschied in der Wirksamkeit der beiden Opioide führen die Studienautoren im Wesentlichen auf die unterschiedliche Pharmakokinetik zurück.

- ple

- Mercadante S et al. J Pain Symptom Manage 2015; online 21. August 